\title{
Inhibition of proliferation and invasion of hepatocellular carcinoma cells by IncRNA-ASLNC02525 silencing and the mechanism
}

\author{
ZI CHEN $^{1}$, DONGWEN XU ${ }^{2}$ and TAO ZHANG ${ }^{2}$ \\ Departments of ${ }^{1}$ Hematology and ${ }^{2}$ Laboratory Medicine, Huashan Hospital, Fudan University, Shanghai 200040, P.R. China
}

Received May 5, 2017; Accepted June 26, 2017

DOI: 10.3892/ijo.2017.4069

\begin{abstract}
One of the most differentially expressed long non-coding RNAs (lncRNAs) that we identified by high throughput screening from liver cancer and para-carcinoma tissues, ASLNC02525, was highly expressed in the tissues and cell lines of liver cancer but not in adjacent tissues or normal hepatic cells. Knockdown of ASLNC02525 in hepatocellular carcinoma cells inhibited the proliferation and invasion. In the process, expression level of transcription factor twist1 (twist-related protein 1) was reduced, but no change at transcription level was observed. According to bioinformatics analysis, ASLNC02525 may play a crucial role in inactivation of regulation of twist1 by hsa-miRNA-489-3p. The mechanism study revealed that ASLNC02525, as an RNA sponge, broke the negative regulation of twist1 by hsa-miRNA-489-3p, and once ASLNC02525 was silenced, the highly expressed hsa-miRNA-489-3p regained its regulation on twist1 and inhibited the proliferation and invasion. The importance of this study lies in shedding light on the potential for lncRNAs to become targets for gene therapy, by demonstrating that lncRNAs can suppress tumor inhibiting activity of miRNAs via breaking regulation of some miRNA target genes.
\end{abstract}

\section{Introduction}

Both miRNAs and lncRNAs play important roles in regulating cellular processes (1). It was found that lncRNA can influence the occurrence and development of tumor by regulating expression of miRNAs, and will cause dysfunction of protein encoding genes if expressed abnormally (2). The expression profile of IncRNAs in a variety of tumor cells differs from that in normal cells, which may contribute to the tumor development (3). The study on the interaction between miRNAs and IncRNAs will revolutionize our knowledge about cell

Correspondence to: Dr Tao Zhang, Department of Laboratory Medicine, Huashan Hospital, Fudan University, 12 Central Urumqi Road, Shanghai 200040, P.R. China

E-mail: shmuzt@126.com

Key words: IncRNA, ASLNC02525, hsa-miRNA-489-3p, twist1, liver cancer, RNA sponge, knockdown structural network and regulatory network, and bring immeasurable scientific and clinical value.

ASLNC02525, is a highly expressed lncRNA in liver cancer that we identified by comparing the expression profile of lncRNAs in clinical liver cancer and adjacent tissues. To elucidate the role of ASLNC02525 in liver cancer, we examined ASLNC02525 levels in three selected liver cancer cell lines, HepG2, QGY-7701 and SMMC-7721, by fluorescent quantitation, and found higher expression of ASLNC02525 in cancer cells than in the normal liver epithelial cells L-02. We knocked down ASLNC02525 in these cell lines via gene interference, which significantly inhibited proliferation and invasion, indicating an oncogene-like role of ASLNC02525. To further explain the tumor-promoting action of ASLNC02525, we silenced ASLNC02525 in HepG2, and screened the differentially expressed proteins, among which one was twist1. We wandered whether ASLNC02525 directly regulates twist1. The results revealed that silencing ASLNC02525 only increased protein level but not mRNA level of twist1. At the same time, RIP experiment proved that ASLNC02525 did not bind to twist1 protein directly. We searched for the miRNAs having potential to bind to 3'-UTR (untranslated regions) of twist1 as well as the ones that theoretically bind to ASLNC02525, and found hsa-miRNA-489-3p, which has one theoretical binding site on 3'-UTR of twist1 and four sites on ASLNC02525. Thus, we progressed to validate whether hsa-miRNA-489-3p is associated with the effect of ASLNC 02525 on twist1 protein.

The twist family of basic helix-loop-helix transcription factors are highly conserved in nucleotide and amino acid sequences, and known for their crucial role in embryonic development $(4,5)$. Nowadays, as one of the hotspots in cancer research, twists have been proved to contribute to the initiation and development of a multitude of cancers (6-10). It can affect a host of signaling pathways, such as PI3K/AKT, STAT3 and ras pathways $(10,11)$, playing a role of an oncogene (12-16).

In the present study, we validated that high expression of ASLNC02525 impaired the negative regulation of twist1 by hsa-miRNA-489-3p like an RNA sponge. The high expression of ASLNC02525 canceled the inhibition of twist 1 by hsa-miRNA-489-3p, and silencing of ASLNC02525 recovered the negative regulation, so the twistl protein was decreased, subsequently inhibiting the proliferation and invasion of liver cancer cells, which provides a novel thought and theoretical basis for gene therapy of liver cancer. 


\section{Materials and methods}

Cell culture. HepG2, QGY-7701, SMMC-7721 and L-02 cells, obtained from the Cell Bank of the Chinese Academy of Sciences (Shanghai, China), were maintained in RPMI-1640 (Invitrogen, Carlsbad, CA, USA) supplemented with $10 \%$ fetal calf serum (FCS; Invitrogen). 293T cells, purchased from the American Type Culture Collection (ATCC; Rockville, MD, USA) were maintained in Dulbecco's minimum essential medium (DMEM) supplemented with 10\% FBS. All these adherent cells were passaged by $0.25 \%$ trypsin digestion (Invitrogen) and incubated in an atmosphere of $5 \% \mathrm{CO}_{2}$ at $3^{\circ} \mathrm{C}$.

\section{Construction of vectors}

Construction of pSIH1-shRNA-ASLNC02525 plasmid. A siRNA sequence complementarily binding to ASLNC02525 was chosen. The target sequences of siRNA (5'-GTCTGTGT CGCCCGTCTGTG-3') are homologous to ASLNC02525, respectively. The oligonucleotide templates of these shRNAs were chemically synthesized and cloned into the linear pSIH1-H1-copGFP shRNA Vector (System Biosciences, Palo Alto, CA, USA) which was obtained through digestion by BamHI and EcoRI (Takara, Dalian, China) and purification by agarose gel electrophoresis. An invalid siRNA sequence (5'-GAAGCCAGATCCAGCTTCC-3') was used as an NC (negative control). Sequencing was used to confirm the vectors constructed (pSIH1-shRNA-ASLNC02525 and pSIH1-NC).

Construction of luciferase reporter vectors. Total RNA was extracted from 293 cells (ATCC), reverse-transcribed into cDNA, and $2 \mu \mathrm{l}$ of the reaction product subsequently was used as a template for PCR. Primers were designed that targeted the 3'-UTR of the twistl gene such that flanking $X b a$ I restriction sites were introduced into the $224 \mathrm{bp}$ (base-pair) PCR product containing the 5'-GATGTCA-3' hsa-miRNA-489-3 target site. The forward and reverse primer sequences were 5'-GCTCTAGACAGCAGGGCCGGAGACCTA-3' and 5'-GC TCTAGAGGTGCCGCTGCCCGTCTGGGAA-3', respectively. PCR reaction conditions were as follows: 35 cycles of a 94 denaturing step for $30 \mathrm{sec}$, a 55 annealing step for $30 \mathrm{sec}$, and a 72 elongation step for $10 \mathrm{sec}$. The PCR product was digested with $\mathrm{XbaI}$ (Takara) and cloned into the pGL3promoter luciferase reporter vector (Promega, Madison, MI, USA) to generate the vector pGL3-Pro-WT-twist. The hsa-miRNA-489-3p target site in the pGL3-Pro-WT-twist vector was mutated from 5'-GATGTCA-3' to 5'-GCAGTAT-3' to construct the mutated reporter vector, pGL3-Pro-MT-twist. The products of all cloning and mutagenesis reactions were confirmed by the DNA sequencing. Endotoxin-free DNA was prepared in all cases. The hsa-miRNA-489-3p mimic (5'-GUGACAUCACAUAUACGGCAGC-3'), the hsa-miRNA-489-3p inhibitor (5'-GCUGCCGUAUAUGUG AUGUCAC-3), and negative control miRNA (miRNA-NC, 5'-UAGAUCGCAGCUUGGAACCACA-3') were all chemically synthesized (Invitrogen).

Lentivirus packaging. One day before the transfection, 293TN cells were seeded into $10-\mathrm{cm}$ dishes (Corning, Inc., Corning NY,USA). A total of $2 \mu \mathrm{g}$ of each pSIH1-shRNA-ASLNC02525 vector or pSIH1-NC and $10 \mathrm{~g}$ pPACK Packaging Plasmid mix
(System Biosciences) were co-transfected using Lipofectamine 2000 (Invitrogen) in accordance with the manufacturer's protocol. The medium was replaced with Dulbecco's modified Eagle's medium (DMEM) plus 1\% fetal bovine serum (FBS). Forty-eight hours later, the supernatant was harvested and then was cleared by centrifugation at $5000 \mathrm{x} \mathrm{g}$ at $4^{\circ} \mathrm{C}$ for $5 \mathrm{~min}$, and passed through a $0.45-\mu \mathrm{m}$ PVDF membrane (Millipore, Billerica, MA, USA). The titer of virus was determined by gradient dilution. The packaged lentiviruses were named as Lv-shRNA-ASLNC02525 and Lv-NC.

Assessment of ASLNC02525, hsa-miRNA-489-3p and twistl protein in liver cancer specimens and liver cancer cell lines. Five pairs of liver cancer and para-carcinoma tissues (Huashan Hospital, Shanghai, China), as well as HepG2, QGY-7701, SMMC-7721 and L-02 cells ( $1 \times 10^{7}$ each), were collected, followed by total RNA extraction and real-time PCR for measurement of ASLNC02525 and hsa-miRNA-489-3p and total protein extraction and western blotting for twist1.

Effect of silencing ASLNC02525 on hsa-miRNA-489-3p and twist1 protein. HepG2, QGY-7701, SMMC-7721 and L-02 in logarithmic phase were seeded to 6 -well plates at $5 \times 10^{5}$ cells/well. One day later, viral solution was added at a MOI of 10. The infection efficiency was evaluated by observing and analyzing the fluorescent mark $72 \mathrm{~h}$ after the infection. In addition, total RNA and protein were isolated from the cells and subjected to real-time PCR and western blotting for ASLNC02525 and twist1 protein, respectively.

Luciferase reporter experiment. We used TargetScan (http:// www.targetscan.org/) to predict whether an hsa-miRNA489-3p binding site exists within the 3'-UTR of human twist1 mRNA (NM_000474.3). The results showed that a seven-base hsa-miRNA-489-3p seed sequence is present in the 3'-UTR of twist 1 mRNA. The same tool was used to predict the binding sites of hsa-miRNA-489-3p on ASLNC02525.

A suspension of 293 cells in logarithmic phase growth was prepared and the number of viable cells counted using a hemocytometer in conjunction with trypan blue staining. The cells were seeded into 6-well plates at a concentration of $2 \times 10^{5}$ cells/well and maintained in DMEM supplemented with $10 \% \mathrm{FCS}$ at $37^{\circ} \mathrm{C}$ for $24 \mathrm{~h}$ in a $5 \% \mathrm{CO}_{2}$ atmosphere. The transfection of plasmid DNA and RNA was performed using Lipofectamine 2000 (Invitrogen). Transfection of cells with pGL-TK (100 ng) served as a reference for luciferase detection. Luciferase activity was measured using the Dual-luciferase reporter assay system (Promega) $48 \mathrm{~h}$ after the transfection.

The experiment to observe the effect of ASLNC02525 depletion on the inhibition of luciferase by hsa-miR489-3p mimics was carried out in HepG2 cells, the plasmid transfection and luciferase activity assay were the same as that used in validation of the target site.

Cellular proliferation assay. Increased proliferation is one indicator in liver cancer cells whether ASLNC02525 knockdown could inhibit proliferation. HepG2, QGY-7701 and SMMC-7721 cells infected with recombinant lentiviruses (Lv-shRNA-ASLNC02525) for $72 \mathrm{~h}$, trypsinized, and seeded into 96 -well plates at a density of $1 \times 10^{4}$ cells/well. The cells 
were cultured under normal conditions and cell viability was examined using Cell Counting kit-8 (CCK-8; Dojindo Laboratories, Inc., Kumamoto, Japan) at 24-, 48- and 72-h timepoints. Briefly, $10 \mu \mathrm{l}$ of CCK-8 solution was added, and the cells were then cultured under normal conditions for an additional $4 \mathrm{~h}$ before the measurement of absorbance at $450 \mathrm{~nm}$.

Cell invasion assay. Cell invasion experiments were performed using the $\mathrm{QCM}^{\mathrm{TM}}$ 24-well Fluorimetric Cell Invasion Assay kit (ECM554; Chemicon International, Temecula, CA, USA) according to the manufacturer's instructions. The kit uses an insert polycarbonate membrane with an $8-\mu \mathrm{m}$ pore size. The insert was coated with a thin layer of EC Matrix ${ }^{\mathrm{TM}}$ that occluded the membrane pores and blocked migration of noninvasive cells. Culture medium $(500 \mu \mathrm{l})$ supplemented with $10 \%$ FBS was used as chemoattractant. Cells that migrated and invaded the underside of the membrane were fixed in $4 \%$ paraformaldehyde. The invading cells were stained by DAPI, and the number was then determined by fluorescence and reported as relative fluorescence units (RFUs).

Examination of effects of ASLNC02525 on twistl and downstream proteins. PI3K, STAT3 and E-cadherin levels in HepG2, QGY-7701 and SMMC-7721 cells were assessed using western blotting $72 \mathrm{~h}$ after the infection with Lv-NC or Lv-shRNA-ASLNC02525.

Real-time PCR. Total RNA was isolated with TRIzol reagent (Invitrogen) according to the manufacturer's instructions and reversely transcribed into cDNA using the M-MLV Reverse Transcriptase (Takara Bio, Inc., Shiga, Japan) and oligo(dT)18 primer (Takara Bio). The following specific primers were used in quantitative PCR of human ASLNC02525 and $\beta$-actin: ASLNC02525: 5'-TTCCAGTGACTCCACGTGC-3' and 5'-AACTTTGGGCCTGTGCCGAAGGGT-3'; and $\beta$-actin: 5'-CCTGTACGCCAACACAGTGC-3' and 5'-ATACTCCTGC TTGCTGATCC-3'. The lengths of amplified products were 219 and $211 \mathrm{bp}$, respectively. Real-time PCR was performed using SYBR Premix Ex Taq ${ }^{\mathrm{TM}}$ kit (Takara) and TP800 System (Takara Bio). cDNA from 200 ng total RNA was used as the template. The PCR reactions was carried out under the following conditions: 40 cycles of denaturation at $95^{\circ} \mathrm{C}$ for $10 \mathrm{sec}$, annealing at $60^{\circ} \mathrm{C}$ for $20 \mathrm{sec}$ and extension at $72^{\circ} \mathrm{C}$ for $20 \mathrm{sec}$. The mRNA levels of twist 1 were normalized using the $\Delta \Delta \mathrm{Ct}$ method, to the expression of an endogenous housekeeping gene, $\beta$-actin.

The expression of hsa-miRNA-489-3p was analyzed with the $2^{-\Delta \Delta C t}$ method. For each sample, triplicate determinations were performed, and mean values were adopted for further calculations. All values were normalized to an endogenous U6 control. The PCR primers for mature hsa-miRNA-489-3p or U6 were designed as follows: hsa-miRNA-489-3p sense, 5'-GTGACATCACATATACGGCAGC-3' and reverse, 5'-GT CGTATCCAGTGCGTGTCGTG-3'; U6 sense, 5'-GTGCTC GCTTCGGCAGCACAT-3' and reverse, 5'-TACCTTGCGA AGTGCTTAAAC-3'.

Detection of protein contents in cells or tissues. The total protein was extracted from the cells using M-PER mammalian protein extraction reagent (Pierce, Rockford, IL, USA) or from
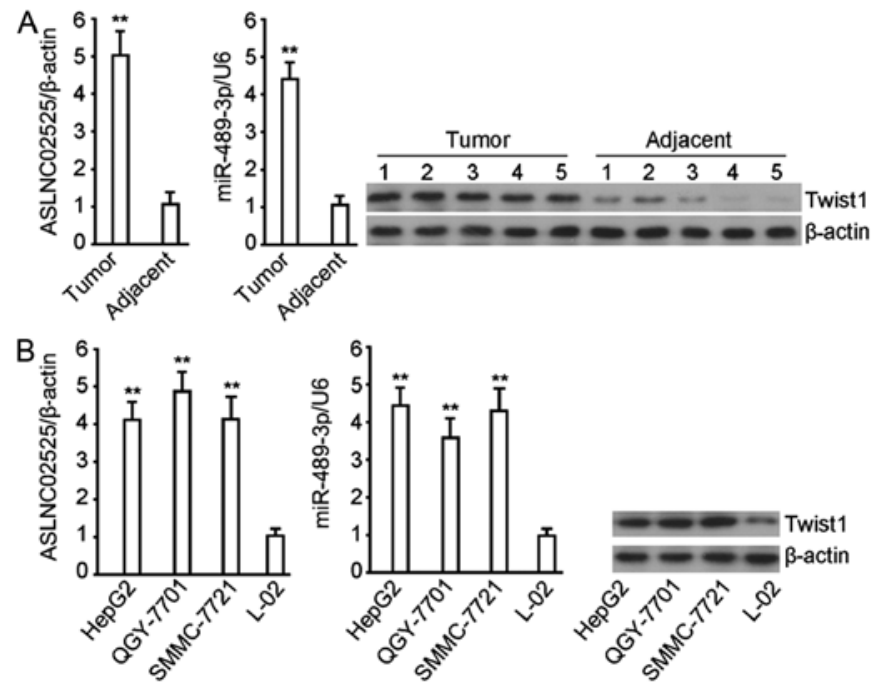

Figure 1. The relative level of ASLNC02525, hsa-miRNA-489-3p and twist1 protein in liver cancer tissues and liver cancer cells. (A) Measurement of ASLNC02525 and hsa-miRNA-489-3p by real-time PCR (left and middle panel) and twist 1 protein by western blotting (right panel) in cancer and adjacent tissues. (B) Measurement of ASLNC02525, hsa-miRNA-489-3p (left and middle panel) and twist1 protein level (right panel) in 3 liver cancer cells (HepG2, QGY-7701 and SMMC-7721) and L-02 cells. $\beta$-actin and U6 was used as internal controls in quantitative analysis of ASLNC02525 and hsa-miRNA-489-3p, and $\beta$-actin was used as a loading control for western blotting. Results are means \pm SD of at least 3 separate experiments. ${ }^{* *} \mathrm{P}<0.01$, when compared to adjacent normal tissue or L-02 cells. For western blotting, representative blots are shown.

tissues using T-PER tissue protein extraction reagent (Pierce). Equal amounts of protein ( $25 \mu \mathrm{g}$ per lane) estimated by a bicinchoninic acid (BCA) protein assay kit (Pierce) were loaded onto (11\%) SDS-PAGE gels and transferred onto nitrocellulose membranes. The blots were probed with a monoclonal antibody against human twist 1 (1:300), PI3K (1:500), STAT3 $(1: 600)$, E-cadherin $(1: 350)$ and $\beta$-actin $(1: 1,200)$ (Santa Cruz Biotechnology, Santa Cruz, CA, USA), followed the secondary HRP-conjugated anti-mouse/rabbit antibody (Santa Cruz Biotechnology). After washing, the bands were detected by chemiluminescence and imaged with X-ray film. $\beta$-actin was used as an endogenous reference for normalization.

Statistical analysis. All data are expressed as mean \pm SD, and were analyzed by the one-way ANOVA. Least significant difference (LSD) was used for multiple comparisons between any two means. P-values $<0.05$ were considered statistically significant. All statistical analyses were performed using SPSS 13.0 software.

\section{Results}

Assessment of ASLNC02525, hsa-miRNA-489-3p and twist1 protein in liver cancer tissues. The expression of twist1 was higher in liver cancer than in the adjacent tissue $(\mathrm{P}<0.01)$ (Fig. 1A), so were ASLNC02525 and hsa-miRNA-489-3p. Compared with normal liver cell line L-02, the three cancer cell lines exhibited higher twist1, as well as ASLNC02525 and hsa-miRNA-489-3p ( $\mathrm{P}<0.01)$ (Fig. 1B). The finding that twist1, a predicted target gene of hsa-miRNA-489-3p, was positively correlated with hsa-miRNA-489-3p in tissue and liver cancer 

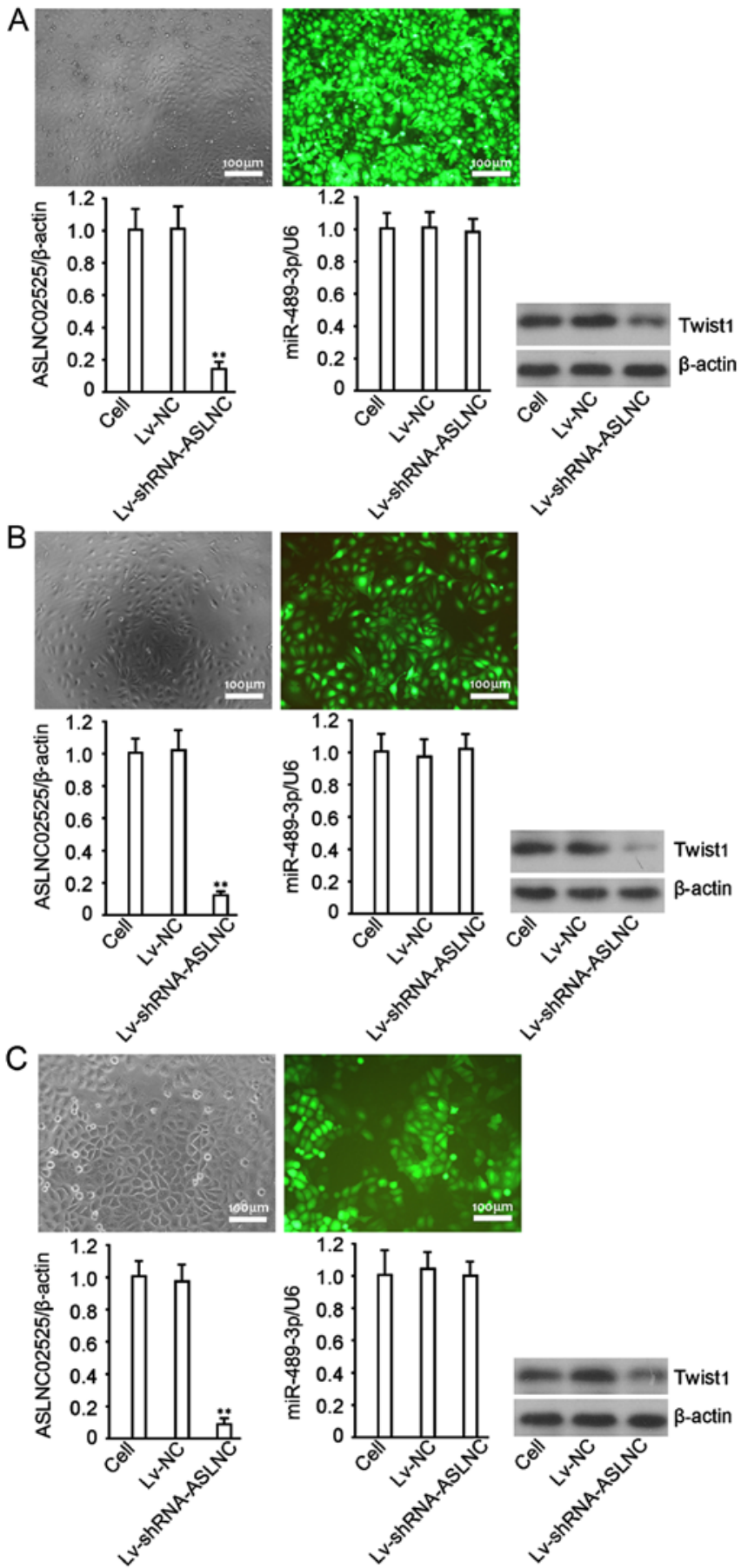

Figure 2. ASLNC02525 depletion decreases twist1 protein but not hsamiRNA-489-3p. (A) HepG2, (B) QGY-7701, (C) SMMC-7721 were infected with Lv-NC or Lv-shRNA-ASLNC02525. RNA and protein were extracted and subjected for measurement of ASLNC02525, hsa-miRNA-489-3p and twistl by real-time PCR and western blotting, respectively, $72 \mathrm{~h}$ later. Upper, cells were infected with Lv-shRNA and GFP was observed under fluorescence microscopy $72 \mathrm{~h}$ later. Lower (left and middle panel): ASLNC02525 and hsa-miRNA-489-3p levels were assessed by quantitative PCR. Data are expressed as mean $\pm \mathrm{SD}$ of at least three independent experiments. ${ }^{* *} \mathrm{P}<0.01$, when compared to control groups. Lower (right panel): effects of ASLNC02525 knockdown on twist1; representative blots are shown.

cells raised the question whether hsa-miRNA-489-3p regulates twist1, which we investigated in following experiments.

Effect of silencing ASLNC02525 via lentiviral approach on hsa-miRNA-489-3p and twistl protein in liver cancer
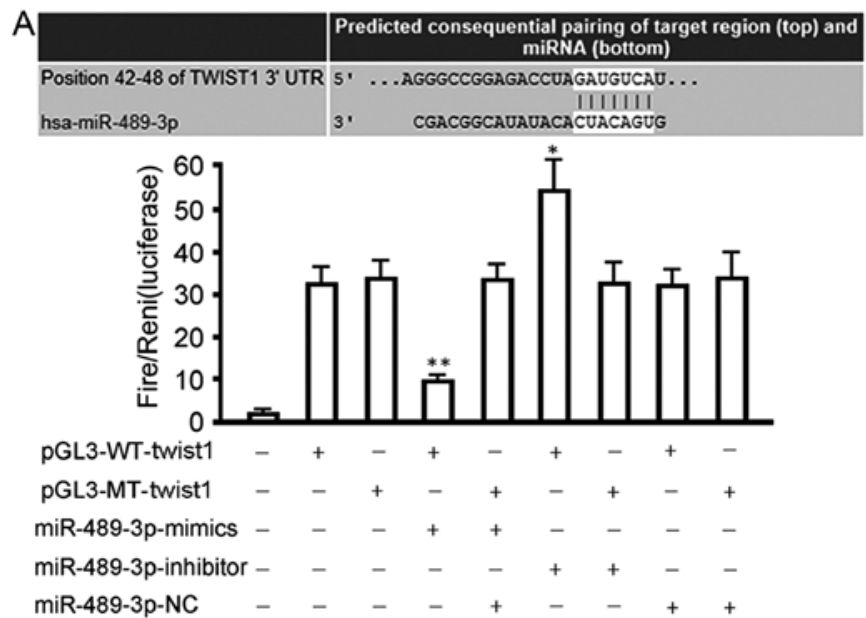

miR-489-3p-NC

B

5:GGCTTTCACTTAGCATGATGTCTCCAAGTCTGTGTCGCCCGTCTGTGTCGCCCAGTGCAT CAGGGTCTCATTCCTTTTGACCGTGGGCGCCGTTCTACGGCCTGGATTCCAGTGCCTGGAC TGGCCATGTTTAGTCTATGCACGCCTCAGTGGATGGACAGGGTTTCCACCTTGCCGCTATG GCGAAGGATGCTGCCGCTATGGCGAAGGATGCTGCCGCTATGGTGAAGGATGCTGCCGCT TTGGCGAAGGATGCTGCCGCTATGGCGAAGGATGATGCCGCTGTGACGACAGCTGCTGCC GCTGTGGCGAAGGATGCTGCCGCTGTGACGACGGATGCTGCCGCTATGATGAAGGATGAT GCCGCTGTGACGACGGATGCTGCCACTATGGCGAAGGATGCTGCCGTGAACTTTGGTGCA CAGGCATCTGTTTGCGTCTTTGCTTTCAATTCCTGTGGGTGGAGCTAGGAGTGGAATTGCT GGGTCACACGGTAACTCTTGGAGGAGCT-3

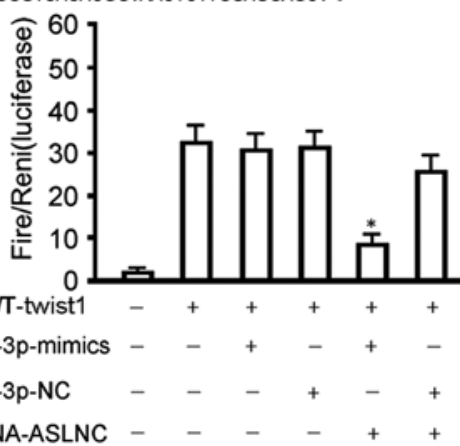

Figure 3. hsa-miRNA-489-3p binds to twist1 3'UTR, which is interfered by ASLNC02525. (A) Cells (293) were transfected with pGL3-WT-twist or pGL3-MT-twist in the presence or absence of miRNA-489-3p-mimic or inhibitor and subjected to luciferase activity assay $48 \mathrm{~h}$ later. Upper panel, predicted binding site of hsa-miRNA-489-3p in 3'-UTR of twist1 gene; lower panel, effects of hsa-miRNA-489-3p on the expression of a luciferase cassette encoding the twist1 3'-UTR. The histogram shows the relative firefly luciferase activity for the different experimental groups. ${ }^{*} \mathrm{P}<0.05$ and ${ }^{* *} \mathrm{P}<0.01$, compared with the group transfected with the same vector but without the miRNA-489-3p mimics or miRNA-489-3p inhibitor. (B) HepG2 cells were transfected with the indicated vectors and subjected to luciferase activity assay $48 \mathrm{~h}$ later. Upper panel, predicted binding site of hsa-miRNA-489-3p in ASLNC02525; lower panel, expression of a luciferase cassette encoding the ASLNC02525. The histogram shows the relative firefly luciferase activity for the different experimental groups. ${ }^{*} \mathrm{P}<0.05$, compared with the group transfected with pGL3-WT-twist and miR-489-3p-mimics. Data are expressed as mean $\pm \mathrm{SD}$ of at least three independent experiments.

cells. Recombinant lentiviruses, Lv-NC and Lv-shRNAASLNC02525, were used to infect HepG2, QGY-7701 and SMMC-7721. Green fluorescent protein (GFP) was detected in most of the cells $72 \mathrm{~h}$ after the infection, and the proportion of GFP-expressing cells suggested that the gene delivery efficiency was $>90 \%$ in all three cell lines (Fig. 2, left panels). ASLNC02525 was significantly decreased by Lv-shRNAASLNC02525 ( $\mathrm{P}<0.05)$ (Fig. 2, middle panels). No change in hsa-miRNA-489-3p was observed, but twist1 protein was significantly decreased $(\mathrm{P}<0.01)$ (Fig. 2 , right panel). The finding is of importance: in the presence of high ASLNC02525 

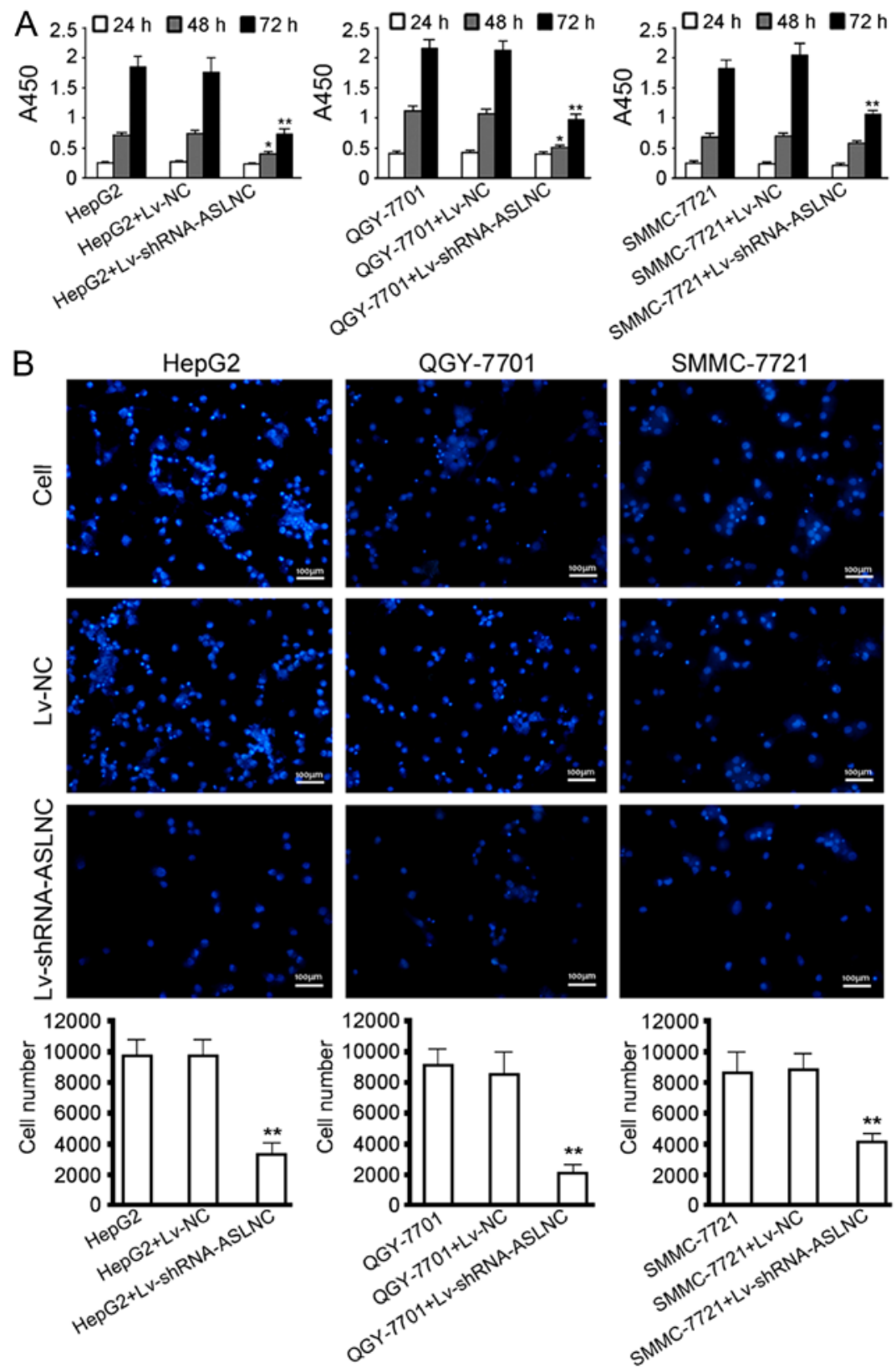

Figure 4. Effects of ASLNC02525 depletion on proliferation and invasion of hepatoma carcinoma cells. (A) HepG2, QGY-7701 and SMMC-7721 were infected with the indicated lentivirus and then seeded to 96 -well plates and subjected to cell vitality assay at the 24,48 and $72 \mathrm{~h}$. (B) Cell invasion assay $48 \mathrm{~h}$ after HepG2, QGY-7701 and SMMC-7721 infected with the indicated lentivirus seeding into the upper chamber of a Transwell. Upper, representative images of cells which passed through the basement membrane, lower, statistics of the numbers of invasive cells. Data are expressed as mean \pm SD of at least three independent experiments. ${ }^{*} \mathrm{P}<0.05 ;{ }^{* *} \mathrm{P}<0.01$, compared with the control groups.

expression, hsa-miRNA-489-3p was not correlated to twist1 protein expression but in the absence of ASLNC02525, hsa-miRNA-489-3p was negatively correlated to twist 1 protein.

Luciferase experiments. Our bioinformatics analysis identified a seven-base hsa-miRNA-489-3p seed sequence in the 3'-UTR of twist1 mRNA (Fig. 3A). We therefore constructed luciferase reporter vectors to verify whether this site represents a valid hsa-miRNA-489-3p target. Reporter vectors were generated that contained the wild-type twist1 3'-UTR or a variant in which the hsa-miRNA-489-3p target site within the 3'-UTR had been mutated. Both reporter constructs expressed luciferase at a high level (Fig. 2A). However, the
miRNA-489-3p mimic significantly inhibited luciferase activity in cells transfected with the reporter vector encoding the wild-type 3 '-UTR $(32.43 \pm 3.69$ vs. $9.01 \pm 1.08 ; \mathrm{P}<0.01)$, while the miRNA-489-3p inhibitor significantly increased luciferase activity in these cells $(33.78 \pm 4.02$ vs. $51.84 \pm 11.02$; $\mathrm{P}<0.05)$. Conversely, in cells transfected with the reporter vector encoding the mutated hsa-miRNA-489-3p target site, neither the miRNA-489-3p mimic nor the miRNA489-3p inhibitor had any observable effect on luciferase activity $(\mathrm{P}>0.05)$. Co-transfection of miRNA-489-3p-NC (nontargeting control) had no effect on the luciferase activity of either of the vectors $(\mathrm{P}>0.05)$. These results verified the presence of an hsa-miR-489-3p target site in the 3'-UTR of twist1 mRNA and demonstrated that binding of hsa-miR-489-3p 


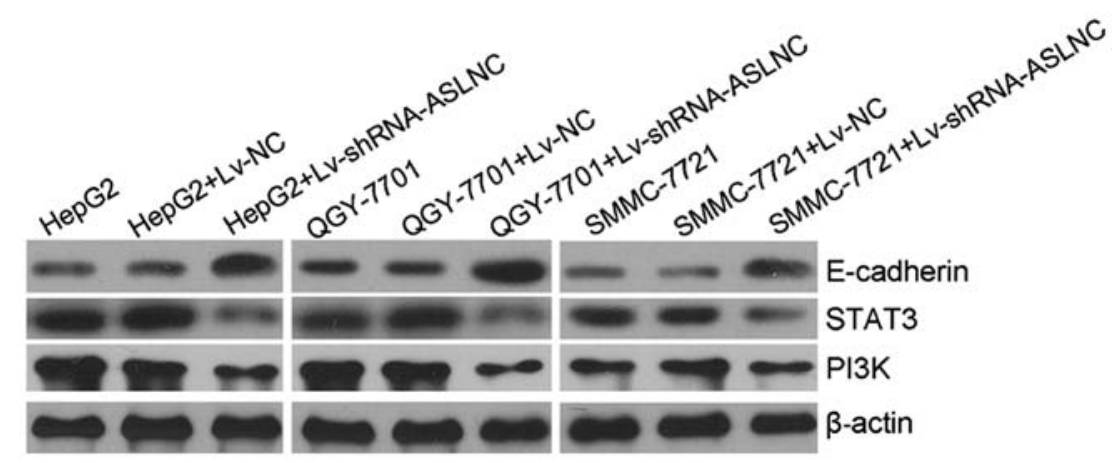

Figure 5. Effects of ASLNC02525 silencing on E-cadherin, STAT3 and PI3K. (Left, middle and right panel) The expression of E-cadherin, STAT3 and PI3K in HepG2, QGY-7701 and SMMC-7721 cells infected with or without Lv-NC or Lv-shRNA-ASLNC02525 $72 \mathrm{~h}$ post infection were detected using western blot analysis. $\beta$-actin as a loading control. Data are representative of at least three independent experiments.

to this target site downregulates twist1 expression. Notably, miRNA-489-3p mimics lost its inhibition on the activity of luciferase expressed by wild-type (WT) luciferase reporter vector in HepG2 cells, and regained the inhibition after ASLNC02525 silencing (Fig. 3B). Taken together, these data suggest that ASLNC02525 overexpression impaired negative regulation of twistl by hsa-miRNA-489-3p.

Effect of ASLNC02525 depletion on proliferation and invasion of hepatoma carcinoma cells. As suggested by cell proliferation assays (Fig. 4A), ASLNC02525 depletion effectively inhibited the proliferation of HepG2, QGY-7701 and SMMC-7721 cells $(\mathrm{P}<0.01) 72 \mathrm{~h}$ after the infection. The invasion assays (Fig. 4B) suggest that ASLNC02525 silencing significantly reduced the invasion of these three cell lines, reflected by decrease in the numbers of cells which passed through the basement membrane $(\mathrm{P}<0.05)$.

Effects of ASLNC02525 silencing on twistl and its downstream proteins in liver cancer cells. We also assessed STAT3, PI3K and E-cadherin in the ASLNC02525-depleted cells. The results (Fig. 5) showed that STAT3 and PI3K were decreased and E-cadherin was increased by ASLNC02525 silencing but not the Lv-NC. Decrease in STAT3 and PI3K is detrimental to cancer and the increase in E-cadherin can inhibit epithelialmesenchymal transition (EMT) and reduce invasion of cancer cells.

\section{Discussion}

lncRNAs, endogenous RNAs longer than 200 nucleotides discovered recently and attracting much interest, have been found to have functions associated with various biological processes via epigenetic modification. Increasingly major studies suggest tangible involvement of lncRNA in cancer. Analysis of chromatin reveals that there are over 1000 IncRNAs in mammals, which are less conserved than the coding RNAs. As an important type of regulators, lncRNAs exert their functions through a variety of ways. Although they were firstly regarded as by-products by RNA polymerase II, or transcriptional noise, recent studies have shown that lncRNAs are associated with multiple biological processes such as chromosome silencing, chromatin modification and transcriptional regulation $(17,18)$.
The interaction between lncRNAs and miRNAs has an important influence on the onset and development of cancer (19). miRNAs are able to regulate lncRNAs in a targeted way: a study has shown that miR-21 targets lncRNA GAS5 in addition to protein coding genes (20). IncRNAs can also affect the onset and the development of cancer by regulating expression of miRNAs. According to existing studies, lncRNAs regulate miRNAs through three ways: i) to competitively combine to 3'-UTR of mRNAs with miRNAs, so as to inhibit negative regulation by miRNAs. Pang et al (21) found that an anti-sense RNA complementary to BACE1 can complement to BACE1 mRNA, competitively inhibiting the negative regulation of BACE1 by miRNA; ii) to regulate target genes by forming pre-miRNAs after RNA splicing and producing specific miRNAs; and iii) to act as endogenous miRNA sponge to suppress miRNA function, so as to affect malignant biological behavior of cancer cells (22). Abnormal lncRNA expression is found in diversified diseases, and lncRNA expression profiles in a variety of tumors are different from normal cells. The proportion of lncRNAs in the total transcripts of genome is far larger than that of encoding RNAs. In addition, lncRNAs play crucial roles in the regulatory network though their interaction with DNA, RNA and proteins. In addition to gene expression regulation, IncRNAs are closely related to evolution of species, embryonic development, metabolism and tumorigenesis. The evidence on involvement of lncRNAs in diseases including cancers will provide basis and target for diagnosis and treatment of diseases. Huang et al (23) found lncRNA-Dreh can inhibit hepatocellular carcinoma metastasis. Gabory et al (24) demonstrated that IncRNA H19 is closely associated with the onset of tumors. Dysregulated expression of lncRNAMALAT1 is also found to link to cancer. Moreover, it is shown that $\operatorname{lncRNAs}$ are related to drug resistance of liver cancers: CUDR InRNA, $2.2 \mathrm{~kb}$ in length, located at 19p13.1, can downregulate caspase- 3 and thus, promote growth, so as to induce drug resistance $(25,26)$.

Human twist gene is on chromosome 7, encoding a transcription factor. It was first identified in Drosophila in 1983, and found to be highly conservative between species: Mouse twist has a $96 \%$ amino acid sequence homology to human twist, and their DNA binding domains are $100 \%$ sequence conserved (4). Twist regulates gene transcription by binding to E-box sequence on gene promoters (5). By inhibiting apoptosis, downregulating p53, a key tumor suppressor 
gene, and promoting angiogenesis, it can promote cancer development. Overexpression of twist results in resistance to apoptosis in cancer cells and increasing expression of twist in implanted MCF-7 breast cancer in mice increased blood flow and vascular permeability (27). Twist also plays a pivotal role in EMT, which is the onset of migration and invasion of a host of cancers. Yang et al (28) argued that as a functional basic helix-loop-helix transcription factor, twist may bind to other helix-loop-helix proteins to form dimers, which in turn activate or inhibit downstream targets, so as to regulate E-cadherin. Therefore, upregulation of twist inhibits E-cadherin, and induces EMT, so as to promote migration and invasion of cancer cells. Furthermore, high expression of twist is also linked to multidrug resistance in cancer cells $(29,30)$.

We screened for differential lncRNAs in several pairs of selected hepatocellular carcinoma and adjacent tissue by using lncRNA chips. ASLNC02525, highly expressed in liver cancers, came into view. We next examined ASLNC02525 in liver cancer tissues and three hepatocellular carcinoma cell lines by fluorescent quantitation, and the results were consistent with the screening, that is, expression of ASLNC02525 in cancer tissues and cells was higher than in para-carcinoma tissue and normal hepatic epithelial cells. We knocked down ASLNC02525 in HepG2, QGY-7701 and SMMC-7721 cells by RNAi, and the results suggest that ASLNC02525 knockdown inhibited the proliferation in these cells. We then sought to explore what protein mediates the effects of ASLNC02525 on proliferation and invasion in these cells. Thus, we subjected normal and ASLNC02525 depleted cells to differential protein screening by protein chips, and found twist1 (data not shown). The silencing of ASLNC02525 in liver cancer cells showed a positive correlation between twist 1 expression and ASLNC02525 knockdown. We proceeded to investigate whether ASLNC02525 depletion downregulates twist1 expression or suppresses its activity to inhibit cancer by RIP-Seq experiment (data not shown), and no twistl mRNA was found in the DNA sequence binding to twist1. The result that protein expression but not transcription of twist1 was affected by ASLNC02525 implied that there may be a post-transcription regulation of twistl changed by ASLNC02525 silencing. Since miRNAs are common post-transcription regulation factor, we supposed that abnormal expression of ASLNC02525 may break some regulation of twist1 by a miRNA.

lncRNAs, as a competing endogenous RNA, interact with miRNAs to regulate target genes, so as to play important roles in the onset and development of cancers $(31,32)$. Therefore, we searched for potential miRNAs which may bind to both ASLNC02525 and twist1 3'UTR, and found hsa-miRNA489-3p, which has, respectively, four and one predictive binding site on ASLNC02525 and twist1 3'UTR. The hypothesis surfaced that high expression of ASLNC02525 impairs the regulation of twist1 by hsa-miRNA-489-3p like an RNA sponge, and results in cellular transformation, and the depletion of ASLNC02525 recovers the negative regulation, so as to suppress twist1, as well as cancer activity.

The experimental results proved our hypothesis: both tissues and cells of liver cancer exhibited high levels of ASLNC02525 and hsa-miRNA-489-3p, and silencing ASLNC02525 inhibited proliferation and invasion of liver cancer cells, and reduced twist 1 protein, but no significant changes in
hsa-miRNA-489-3p was observed, indicating twist1 expression inhibition may mediate the suppression of liver cancer cells by ASLNC02525 silencing. The luciferase reporter assay showed that hsa-miRNA-489-3p bound to twist1 3'UTR via its seed site and inhibited protein translation, which was impaired by ASLNC02525 overexpression. In addition, knockdown ASLNC02525 recovered the negative regulation of twist1 by hsa-miRNA-489-3p. Although we proposed a preliminary explanation for suppression of liver cancer by ASLNC02525 depletion, it requires profound future study. In general, special structure is required for competitive binding of lncRNA, so our next step is to establish the structure of ASLNC02525 and the binding of its four predicted sites to hsa-miRNA-489-3p, as well as whether the inhibition is prevalent.

The importance of the study lies in providing some insight into the potential for IncRNA as a target for gene therapy, by demonstrating that lncRNA can suppress tumor inhibiting activity of miRNAs via interrupting the regulation of some miRNA target genes.

\section{Acknowledgements}

This work was supported by the National Clinical Key Subject, the National Natural Science Foundation of China (grant nos. 81072070, 81471241, 81171133, and 81271375) and the Natural Science Foundation of Shanghai (grant no. 15ZR1405600).

\section{References}

1. Abdelmohsen $\mathrm{K}$ and Gorospe M: Noncoding RNA control of cellular senescence. Wiley Interdiscip Rev RNA 6: 615-629, 2015.

2. Alaei-Mahabadi B and Larsson E: Limited evidence for evolutionarily conserved targeting of long non-coding RNAs by microRNAs. Silence 4: 4, 2013.

3. Schmitt AM and Chang HY: Long noncoding RNAs in cancer pathways. Cancer Cell 29: 452-463, 2016.

4. Firulli AB and Conway SJ: Phosphoregulation of Twist1 provides a mechanism of cell fate control. Curr Med Chem 15: 2641-2647, 2008.

5. Atfi A and Baron R: p53 brings a new twist to the Smad signaling network. Sci Signal 1: pe33, 2008.

6. Barsky D and Venclovas C: DNA sliding clamps: Just the right twist to load onto DNA. Curr Biol 15: R989-R992, 2005.

7. Bohlius J, Tonia T and Schwarzer G: Twist and shout: One decade of meta-analyses of erythropoiesis-stimulating agents in cancer patients. Acta Haematol 125: 55-67, 2011.

8. Ai L, Kim WJ, Alpay M, Tang M, Pardo CE, Hatakeyama S, May WS, Kladde MP, Heldermon CD, Siegel EM, et al: TRIM29 suppresses TWIST1 and invasive breast cancer behavior. Cancer Res 74: 4875-4887, 2014.

9. Allaya N, Khabir A, Sallemi-Boudawara T, Sellami N, Daoud J, Ghorbel A, Frikha M, Gargouri A, Mokdad-Gargouri R and Ayadi W: Over-expression of miR-10b in NPC patients: Correlation with LMP1 and Twist1. Tumour Biol 36: 3807-3814, 2015.

10. Ansieau S, Bastid J, Doreau A, Morel AP, Bouchet BP, Thomas C, Fauvet F, Puisieux I, Doglioni C, Piccinin S, et al: Induction of EMT by twist proteins as a collateral effect of tumor-promoting inactivation of premature senescence. Cancer Cell 14: 79-89, 2008.

11. Fu J, Qin L, He T, Qin J, Hong J, Wong J, Liao L and $\mathrm{Xu} \mathrm{J}$ : The TWIST/Mi2/NuRD protein complex and its essential role in cancer metastasis. Cell Res 21: 275-289, 2011.

12. Grzegrzolka J, Biala M, Wojtyra P, Kobierzycki C, Olbromski M, Gomulkiewicz A, Piotrowska A, Rys J, Podhorska-Okolow M and Dziegiel P: Expression of EMT markers SLUG and TWIST in breast cancer. Anticancer Res 35: 3961-3968, 2015. 
13. Ji H, Lu HW, Li YM, Lu L, Wang JL, Zhang YF and Shang H: Twist promotes invasion and cisplatin resistance in pancreatic cancer cells through growth differentiation factor 15 . Mol Med Rep 12: 3841-3848, 2015.

14. Zhang H, Gong J, Kong D and Liu HY: Anti-proliferation effects of Twist gene silencing in gastric cancer SGC7901 cells. World J Gastroenterol 21: 2926-2936, 2015.

15. Kim K, Park EY, Yoon MS, Suh DS, Kim KH, Lee JH, Shin DH, Kim JY, Sol MY and Choi KU: The role of TWIST in ovarian epithelial cancers. Korean J Pathol 48: 283-291, 2014.

16. Han Z, Liu L, Liu Y and Li S: Sirtuin SIRT6 suppresses cell proliferation through inhibition of Twist 1 expression in non-small cell lung cancer. Int J Clin Exp Pathol 7: 4774-4781, 2014.

17. Ponting CP, Oliver PL and Reik W: Evolution and functions of long noncoding RNAs. Cell 136: 629-641, 2009.

18. Bergmann $\mathrm{JH}$ and Spector DL: Long non-coding RNAs: Modulators of nuclear structure and function. Curr Opin Cell Biol 26: 10-18, 2014

19. Deng G and Sui G: Noncoding RNA in oncogenesis: A new era of identifying key players. Int J Mol Sci 14: 18319-18349, 2013.

20. Pickard MR and Williams GT: Molecular and cellular mechanisms of action of tumour suppressor GAS5 lncRNA. Genes (Basel) 6: 484-499, 2015.

21. Pang M, Xing C, Adams N, Rodriguez-Uribe L, Hughs SE, Hanson SF and Zhang J: Comparative expression of miRNA genes and miRNA-based AFLP marker analysis in cultivated tetraploid cottons. J Plant Physiol 168: 824-830, 2011.

22. Brodersen P, Sakvarelidze-Achard L, Schaller H, Khafif M, Schott G, Bendahmane A and Voinnet O: Isoprenoid biosynthesis is required for miRNA function and affects membrane association of ARGONAUTE 1 in Arabidopsis. Proc Natl Acad Sci USA 109: 1778-1783, 2012.

23. Huang JF, Guo YJ, Zhao CX, Yuan SX, Wang Y, Tang GN, Zhou WP and Sun SH: Hepatitis B virus X protein (HBx)-related long noncoding RNA (lncRNA) down-regulated expression by HBx (Dreh) inhibits hepatocellular carcinoma metastasis by targeting the intermediate filament protein vimentin. Hepatology 57: 1882-1892, 2013.
24. Gabory A, Ripoche MA, Le Digarcher A, Watrin F, Ziyyat A, Forné T, Jammes H, Ainscough JF, Surani MA, Journot L, et al: $\mathrm{H} 19$ acts as a trans regulator of the imprinted gene network controlling growth in mice. Development 136: 3413-3421, 2009.

25. Lai MC, Yang Z, Zhou L, Zhu QQ, Xie HY, Zhang F, Wu LM, Chen LM and Zheng SS: Long non-coding RNA MALAT-1 overexpression predicts tumor recurrence of hepatocellular carcinoma after liver transplantation. Med Oncol 29: 1810-1816, 2012.

26. Tsang WP, Wong TW, Cheung AH, Co CN and Kwok TT: Induction of drug resistance and transformation in human cancer cells by the noncoding RNA CUDR. RNA 13: 890-898, 2007.

27. Mironchik Y, Winnard PT Jr, Vesuna F, Kato Y, Wildes F, Pathak AP, Kominsky S, Artemov D, Bhujwalla Z, Van Diest P, et al: Twist overexpression induces in vivo angiogenesis and correlates with chromosomal instability in breast cancer. Cancer Res 65: 10801-10809, 2005.

28. Yang J, Mani SA, Donaher JL, Ramaswamy S, Itzykson RA, Come C, Savagner P, Gitelman I, Richardson A and Weinberg RA: Twist, a master regulator of morphogenesis, plays an essential role in tumor metastasis. Cell 117: 927-939, 2004.

29. Al Saleh S1, Sharaf LH and Luqmani YA: Signalling pathways involved in endocrine resistance in breast cancer and associations with epithelial to mesenchymal transition (Review). Int $\mathbf{J}$ Oncol 38: 1197-1217, 2011.

30. Chen HF, Huang CH, Liu CJ, Hung JJ, Hsu CC, Teng SC and Wu KJ: Twist 1 induces endothelial differentiation of tumour cells through the Jagged1-KLF4 axis. Nat Commun 5: 4697, 2014.

31. Chen L, Wang W, Cao L, Li Z3 and Wang X: Long non-coding RNA CCAT1 acts as a competing endogenous RNA to regulate cell growth and differentiation in acute myeloid leukemia. Mol Cells 39: 330-336, 2016.

32. Karreth FA and Pandolfi PP: ceRNA cross-talk in cancer: When ce-bling rivalries go awry. Cancer Discov 3: 1113-1121, 2013. 